\title{
Prevalence of multidrug resistance in human pathogenic Staphylococcus aureus and their sensitivity to Allamanda cathartica L. leaf extract
}

\author{
Md. Al Nayem Chowdhury ${ }^{1}$, Md. Nazmul Hossain ${ }^{1}$, Md. Mahbubur Rahman², ${ }^{*}$ Md. Ashrafuzzaman ${ }^{1}$ \\ ${ }^{1}$ Department of Genetic Engineering and Biotechnology, Shahjalal University of Science and Technology, Sylhet-3114, Bangladesh \\ ${ }^{2}$ Department of Biotechnology, Bangabandhu Sheikh Mujibur Rahman Agricultural University, Gazipur-1706, Bangladesh
}

\begin{abstract}
Staphylococcus aureus is one of the major pathogen responsible for skin infection, urinary tract infection (UTI) and endocarditis in human. The study was performed to determine the prevalence of multidrug resistant S. aureus in human clinical sample and to evaluate their sensitivity to Allamanda cathartica L. leaf extract. A total of 12 isolates were identified belongs to S. aureus by performing several physiological and biochemical tests. The isolates exhibited highest resistant (75\%) to streptomycin and lowest (33.33\%) against co-trimoxazole followed by disc diffusion assay of eight antibiotics tested. The other four antibiotics such as azithromycin, chloramphenicol, gentamycin and erythromycin exhibited 50 to $66.67 \%$ resistant to present isolates. Here we found that $75 \%$ of $S$. aureus isolates were multidrug-resistant (MDR). The crude leaf extract of $A$. cathartica L. found to possess antibacterial properties at the rate of $83.33 \%$ against $S$. aureus isolates with $12-22 \mathrm{~mm}$ zone of inhibition. Results of TLC states that Benzene : Ethyl acetate (1:1) solvent system was more effective for initial separation of compound from crude leaf extract resulted three distinct bands with different $R_{\mathrm{f}}$ values ranging from 0.53 to 0.89 . The result of this study refers that $A$. cathartica $L$. leaf extract would be useful to develop effective drugs that would reduce the higher prevalence of multidrug resistance S. aureus causing clinical infection in human.
\end{abstract}

Key Words: Staphylococcus aureus, biochemical identification, antibiogram, disk diffusion assay, leaf extract, TLC.

\section{INTRODUCTION}

Staphylococcus aureus is a gram positive, round shaped, small, non-motile bacteria belongs to the family Staphylococcaceae. It is the leading bacteria in the normal flora of humans especially, in the skin and nasal vestibule and causes a variety of clinical infections including septicemia, pneumonia, osteomyelitis, Endocarditis, UTI, wound sepsis, septic arthritis, and post-surgical toxic shock syndrome with substantial rates of morbidity and mortality (Boyce, 1997; Engemann, 2003). Recently, this organism is being acquired resistance to different antibiotics and development of resistance to antimicrobial agents by Staphylococci is still frequently associated with hospital and community acquired infections (Locksley et al., 1982). Strains of $S$. aureus resistant to $\beta$-lactam antibiotics are known as methicillin-resistant $S$. aureus (MRSA). It is also called multidrug-resistant (MDR) $S$. aureus. The emergence of Multi- drug resistant bacteria is a major problem for treatment of diseases using antibiotics. In recent years it has been reported that the clinical administration of antibiotics, against the pathogenic bacteria be gradually prohibited due to emergence of MDR bacterial strains including S. aureus. (Kumar et al., 2010; Akindele et al., 2010; Hoerlle and Brandelli, 2010, Efuntoye et al., 2011) As a result, searching of alternative and effective medicine against MDR pathogen has become an important concern, all over the world. On the other hand, herbal medicine has been used for the medication of different bacterial disease and and many plant extracts and plant products have been reported to be valuable antimicrobial agent against human pathogens (Maity et al., 2009; Arya et el., 2010; Fuad et al., 2012) A. cathartica

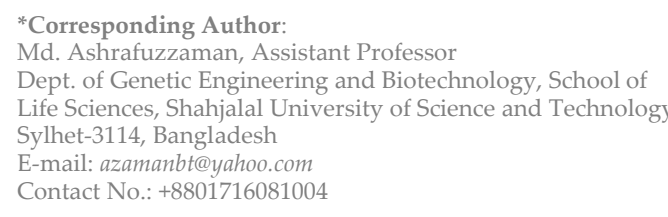

*Corresponding Author:

ylhet-3114, Banglades

Contact No.: +8801716081004

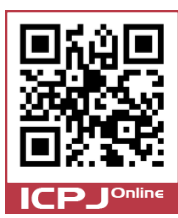

belongs to family Apocynaceae is notable for its medicinal properties. It has been found that the plant extract contain "allamandin", a toxic iridoid lactone and milky sap having antibacterial and possibly anticancer properties (Liogier, 1995). Although the antibacterial effects of $A$. cathartica to different human pathogen have been studied by the previous researchers but the information especially against MDR S. aureus is still not sufficient. As a result, the present study has been carried out to investigate prevalence of MDR S. aureus in human clinical sample and to evaluate the antibacterial effects of $A$. cathartica leaf extract against the MDR S. aureus.

\section{MATERIALS AND METHODS}

Collection of bacterial isolates

A total of 25 presumptive clinical isolates of $S$. aureus were collected from patient suffering Skin infection, Urinary Tract infection (UTI) and Endocarditis, during February 2012, from Popular Diagnostic Center, Sylhet, Bangladesh and aseptically transferred to Laboratory of the Department of Genetic Engineering and Biotechnology, Shahjalal University of Science and Technology for present studies. The isolates were collected with the proper concern of the authority of the diagnostic center as well as permission form each individual patient. Source of the bacterial isolates with information of the patients were recorded (table 1). The isolates were cultured on Nutrient Agar (NA) medium and incubated at $37^{\circ} \mathrm{C}$ for 24 hours. Individual colonies were separated from the plates on the basis of color, shape and size and sub-cultured on the relevant media to obtain pure culture. Then the individual isolates were subjected to biochemical identification.

Identification of bacteria from human clinical sample For the identification of bacterial isolates, several morphological, physiological and biochemical tests were conducted. Morphological appearance of colonies of all isolates was observed after $24 \mathrm{~h}$ incubation (shape, size and color) and subjected to gram staining. The tests 
Table 1: Presumptive $S$. aureus isolates with their source and patient background.

\begin{tabular}{ccccc}
\hline Isolates & \multirow{2}{*}{ Same } & Source & \multicolumn{2}{c}{ Patient Background } \\
\cline { 3 - 5 } & & Disease of the patient & Sex & Age (year) \\
\hline S1 & Puss & Skin infection & Female & 28 \\
S2 & Blood & Endocarditis & Male & 43 \\
S3 & Puss & Skin infection & Male & 37 \\
S4 & Puss & Skin infection & Male & 43 \\
S5 & Puss & Skin infection & Female & 37 \\
S6 & Blood & Endocarditis & Male & 43 \\
S7 & Urine & UTI & Female & 45 \\
S8 & Puss & Skin infection & Male & 29 \\
S9 & Urine & UTI & Female & 51 \\
S10 & Blood & Endocarditis & Male & 39 \\
S11 & Puss & Skin infection & Male & 56 \\
S12 & Urine & UTI & Female & 38 \\
\hline
\end{tabular}

included motility, oxidase activity, catalase test, acid production from glucose, oxidation-fermentation (OF) Voges-Proskauer Test (VP) and Hydrogen Sulfide Production etc. All of the bacterial isolates were identified up to genus level following the Bergey's Manual of Determinative Bacteriology (Bergey and John, 1994).

\section{Antibiogram profiling of bacterial isolates}

Antibiogram assay of present isolates were determined by disc diffusion assay using commercial antibiotic discs followed by Rahman and Hossain (2010). The individual isolates were cultured into nutrient broth and incubated at $37^{\circ} \mathrm{C}$ for 24 hours. Fifty micro liter of individual broth culture was dropped on the NA plate with micropipette. The broth on the plate was spread aseptically by a sterile 'L' shaped glass rod. Eight commercial antibiotics were used in the present study, viz. chloramphenicol $(25 \mu \mathrm{g} / \mathrm{disc})$, azithromycin $(15 \mu \mathrm{g} / \mathrm{disc})$, erythromycin (15 $\mu \mathrm{g} / \mathrm{disc})$, streptomycin $(10 \mu \mathrm{g} / \mathrm{disc})$, co-trimoxazole $(25 \mu \mathrm{g} /$ disc), ciprofloxacin ( $5 \mu \mathrm{g} /$ disc), cephradine $(30 \mu \mathrm{g} / \mathrm{disc})$, centamicin $(10 \mu \mathrm{g} / \mathrm{disc})$. The plates were again incubated at $37^{\circ} \mathrm{C}$ for $24 \mathrm{~h}$. After incubation, each plate was examined for the determination of diameter of zone of inhibition by the antibiotics in millimeter and compared with British Society for Antimicrobial Chemotherapy (BSAC) guideline for antimicrobial susceptibility testing (Andrews, 2012) to determine S. aureus isolates as resistant, intermediate or sensitive.

\section{Antibacterial activity}

The filter paper disk diffusion assay was performed to determine the antibacterial activity of leaf extract of $A$. cathartica L. leaf extract followed by Sharmeen et al. 2012. The $24 \mathrm{~h}$ incubated broth culture of individual bacterial isolates were spread aseptically by ' $L$ ' shaped glass rod and subjected to prepared filter paper disc of A. cathartica leaf extract. The plates then incubated at $37^{\circ} \mathrm{C}$ for

Table 3: Antibiogram profile of S. aureus isolates to different antibiotic disc used.

\begin{tabular}{ccccc}
\hline Name of & Conc. & \multicolumn{3}{c}{ Antibiogram of S. aureus isolates (\%) } \\
\cline { 3 - 5 } Antibiotic & $(\boldsymbol{\mu g} /$ disc) & Resistant & Intermediate & Sensitive \\
\hline Chloramphenicol & 25 & 50 & 16.67 & 33.33 \\
Azithromycin & 15 & 50 & - & 50 \\
Erythromycin & 15 & 66.67 & 25 & 8.33 \\
Streptomycin & 10 & 75 & 25 & - \\
Co-Trimoxazole & 25 & 33.33 & 16.67 & 50 \\
Ciprofloxacin & 5 & 41.67 & 8.33 & 50 \\
Cephradine & 30 & 41.67 & - & 58.33 \\
Gentamicin & 10 & 58.33 & 25 & 16.67 \\
\hline
\end{tabular}

Table 2: Physiological and biochemical characteristics of $S$. aureus.

\begin{tabular}{lc}
\hline Tests & Characteristics \\
\hline Gram stain & + \\
Motility & - \\
Grow Aerobically & + \\
Grow Anaerobically & + \\
O-F test & Fermentative \\
Utilization of Sugars:- & + \\
$\quad$ Glucose & + \\
$\quad$ Lactose & + \\
$\quad$ Mannitol & + \\
Oxidase test & - \\
Catalase test & + \\
MR test & + \\
VP test & + \\
Indole test & - \\
H2S production & + \\
Citrate test & + \\
Urease test & + \\
\hline
\end{tabular}

$+=$ positive, $-=$ negative

overnight and antibacterial activity was determined by measuring the diameter of zone of inhibition with a millimeter scale.

\section{Thin layer chromatography}

The leaf extract of $A$. cathartica $L$. were subjected to thin layer chromatography (TLC) with three different eluting solvents for the effective separation of antimicrobial compounds (Masuduzzaman et al., 2008). TLC plates were removed from hot air oven and sample extract spotted on the activated silica gel allowing capillary action of solvent up the plate until approximately $1 \mathrm{~cm}$ from the end and allowed for air drying. $R_{f}$ value of the compound was measured as the ratio of mobility for bioactive compound to the total length of run. The TLC plates were stained with iodine vapor (Kruiswijk, 2005) and observed under bright light and the separated spots were marked.

\section{RESULTS}

Identification of Staphylococcus aureus isolates

Among 25 isolates a total of 12 isolates were identified belong to S. aureus on the basis of their morphological, physiological and biochemical characteristics. All of the isolates were Gram negative, round shaped, non-motile, catalase positive, oxidase negative, able to utilize sugar (glucose, lactose, sucrose manitol), Methyl-Red (MR) positive Urease positive, Citrate positive and $\mathrm{H}_{2} \mathrm{~S}$ positive (table 2).

Table 4: Sensitivity of S. aureus isolates to Allamanda cathartica L. leaf extracts.

\begin{tabular}{cc}
\hline Isolates Name & Zone of inhibition $(\mathbf{m m})$ \\
\hline S1 & 22 \\
S2 & 18 \\
S3 & 21 \\
S4 & - \\
S5 & 19 \\
S6 & 19 \\
S7 & - \\
S8 & 20 \\
S9 & 17 \\
S10 & 21 \\
S11 & - \\
S12 & 18 \\
\hline
\end{tabular}




\section{Antibiogram profiling of bacterial isolates}

In the present study, the $S$. aureus isolates found to be variable in their resistance pattern against eight antibiotic

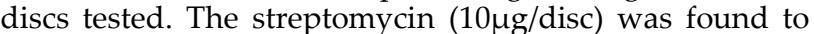
be highest resistant $(75 \%)$ but co-trimoxazole $(5 \mu \mathrm{g} / \mathrm{disc})$ exhibited lowest $(33.33 \%)$ to present isolates. The antibiogram profiles of $S$. aureus isolates to different antibiotics are given in table 3. Other four antibiotics such

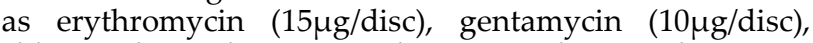

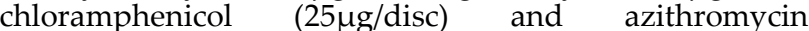
$(15 \mu \mathrm{g} / \mathrm{disc})$ were notably resistant $(50-66.67 \%)$ against $S$.

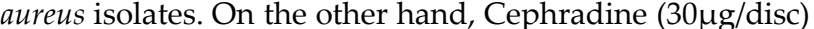
showed as maximum $58 \%$ sensitive to present isolates and

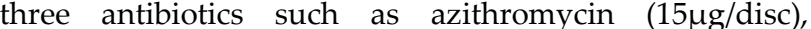

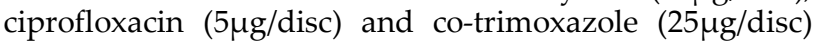
were $50 \%$ sensitive. Here we found that $75 \%$ of S. aureus isolates were resistant to at least two antibiotics and determined as multidrug resistant (MDR) organism.

\section{Sensitivity study}

In this study $83.33 \%$ S. aureus were found to be sensitive to $A$. cathartica leaf extracts. The leaf extract exhibited inhibition zone, ranging from $12-22 \mathrm{~mm}$ followed filter paper disc diffusion assay. Except the two isolates of $S$. aureus (S4 and S11), all of the isolates were found to be sensitive to the leaf extract (table 4).

\section{Initial separation of $A$. cathartica $L$. leaf extract by Thin Layer Chromatography (TLC)}

Compounds initially separated from $A$. cathartica $L$. leaf extracts by TLC were eluted by different solvents which were developed by iodine vapor. The experiment revealed that Benzene : Ethyl acetate (1:1) solvent systems exhibited most effective separation with three bands and their $R_{f}$ value ranges from 0.53 to 0.89 . On the other hand, solvent system Hexane : Benzene(1:1) gave two bands with $\mathrm{R}_{\mathrm{f}}$ values 0.68 and 0.84 . Hexane individually showed least effective separation with only one band of $R_{f}$ value 0.68 (table 5)

\section{DISCUSSION}

The infections, caused by Staphylococcus aureus are quite common all over the world and the emergence of antibiotic-resistant forms of pathogenic $S$. aureus is a worldwide problem in clinical medicine. Here, we collected a total of 25 isolates from clinical patients (table 1) and finally 12 isolates have been identified as $S$. aureus followed by several physiological and biochemical tests. Previously, Similar tests were used by the by the researchers (Silva et al., 2000; Singh and Prakash, 2008; Narmeen and Jubrael, 2009) for the identification of $S$. aureus. In this study, we found that the streptomycin $(10 \mu \mathrm{g} / \mathrm{disc})$ and erythromycin $(15 \mu \mathrm{g} / \mathrm{disc})$ were highly resistant and it was assumed that these drugs would not be effective for medication against $S$. aureus. Although cephradine $(30 \mu \mathrm{g} / \mathrm{disc})$ was maximum $(58 \%)$ sensitive to present isolates but it was also less desirable for the disease treatment. In this study the $75 \%$ of isolates found as multidrug resistant (MDR) and refers that the rate of emergence of MDR S. aureus are very frequent in clinical patient. Recent studies also suggests that emergence of multidrug resistant $S$. aureus are increasing at alarming rate to different antibiotics like streptomycin, erythromycin, gentamicin, chloramphenicol and azithromycin (Hoerlle and Brandelli, 2009; Akindele et al., 2010; Efuntoye et al., 2011; Kumar et al., 2011). Hence, these types of antibiotics would be quite ineffective in the future treatment of infection caused by $S$. aureus. So search for
Table 5: Rf values of different spots in TLC analysis using different solvent systems.

\begin{tabular}{lcl}
\hline Solvent System & $\begin{array}{c}\text { Number } \\
\text { of bands }\end{array}$ & $\begin{array}{l}R_{\mathrm{f}} \text { values of different } \\
\text { spots found }\end{array}$ \\
\hline Benzene : Ethyl acetate (1:1) & 3 & $0.53,0.84,0.89$ \\
Hexane & 1 & 0.68 \\
Hexane : Benzene (1:1) & 2 & $0.68,0.84$ \\
\hline
\end{tabular}

alternative and novel therapeutics is a must at this moment. On the other hand, most of the S. aureus isolates which were multidrug resistant (MDR) in antibiogram profiling but exhibited sensitive to $A$. cathartica $L$. leaf extracts (table 4). Medicinal plants have been used as traditional medication from the ancient times and their antibacterial properties has been reported against several human pathogens in recent research (Khan et al., 2007; Maity et al., 2009). Some recent reports are also available mentioning that the herbal extract can potentially inhibit multidrug resistant human pathogen (Fuad et al., 2012; Sharmeen et al., 2012). Several researchers also found that of $A$. cathartica $L$. leaf extract were able to kill human bacterial pathogen including $S$. aureus (Jeyachandran et al., 2010; Islam et al., 2010). Therefore, A. cathartica L. leaf extract may be useful to reduce the prevalence of multidrug resistant $S$. aureus. The crude leaf extract of $A$. cathartica $L$. was subjected to initial separation by TLC where Benzene : Ethyl acetate (1:1) solvent systems exhibited most effective separation with three bands. However, it was our primary attempt to separate active single compound from the crude leaf extract but to identify and characterize the bioactive compound more preciously, the crude leaf extract of $A$. cathartica $L$. can be further subjected to other chromatographic techniques (Mišan et al., 2011; Sasidharan et al., 2011; Patra et al., 2012).

\section{CONCLUSION}

The present study revealed that, the prevalence of multidrug resistant $S$. aureus is emerging at an alarming rate. As a result, most of the antibiotics are quite ineffective for infection control. On the other hand, A. cathartica $L$. leaf extract was able to poses antibacterial effect including multidrug resistant (MDR) isolates. Therefore, potential antimicrobial drugs may be obtained from Allaromanda cathartica $L$. leaf extract against $S$. aureus associated infection in human.

\section{REFERENCES}

Akindele, A.A. Adewuyi, I.K, Adefioye, O.A. Adedokun S.A., Olaolu, A.O. (2010). Antibiogram and Beta-Lactamase Production of Staphylo coccus aureus Isolates from Different Human Clinical Specimens in a Tertiary Health Institution in Ile-ife, Nigeria. American-Eurasian Journal of Scientific Research, 5, (4), 230-233. [Link]

Andrews, J. (2012). BSAC Methods for Antimicrobial Susceptibility Testing. In British Society for Antimicrobial Chemotherapy (BSAC). 11: pp 1-90. [DOI]

Arya,V., Yadav, S., Kumar, S., Yadav J.P. (2010). Antimicrobial activity of Cassia occidentalis L (Leaf) against various human pathogenic microbes. Life Sciences and Medicine Research, 9 (1) 1-11. [Link]

Berge, A.C.B., Atwill, E.R., Sischo, W.M. (2005). Animal and farm influences on the dynamic of antibiotic resistance in faecal Eschrichia coli in young dairy calves. Preventive Veterinary Medicine, 69, 25-38. [DOI]

Bergey, D.H., John, G.H. (1994). Bergey's manual of determinative biology, Chapter 4, (9th ed., pp. 181-186) William and Wilkins. [Link]

Boyce, J.M. (1997). Epidemiology and prevention of nosocomial infections. Biochemical Characteristics of Typical and Atypical Staphylococcus Aureus in Mastitic Milk And Environmental Samples Of Brazilian Dairy Farms. The staphylococci in human disease, (pp: 309-329). [DOI] 
De-Silva W.P., Destro, M.T., Landgraf, M.,. Franco, B.D.G.M. (2000) Biochemical characteristics of typical and atypical Staphylococcus aureus in mastitic milk and environmental samples of Brazilian dairy farms. Brazilian Journal of Microbiology, 31,103-106. [DOI]

Efuntoye, M.O., Mabekoje, O.O., Adekoya, F.A. (2011). Biochemical, enterotoxigenicity and antibiogram profiles of Staphylococcus aureus isolated from intestines of snails. Journal of Microbiology and Antimicrobials, 3, (3), 47-50. [Link]

Engemann, J.J., Carmeli, Y., Cosgrove, S.E., Fowler, V.G., Bronstein, M.Z., Trivette, S.L., Briggs, J.P., Sexton, D.J., Kaye, K.S. (2003). Adverse clinical and economic outcomes attributable to methicillin resistance among patients with Staphylococcus aureus surgical site infection. Clinical Infectious Diseasee, 36, 592-598. [DOI]

Fuad, M.M.H., Ferdowsy, H., Hossain, M.N., Foysal, M.J., Rahman, M.M. (2012). In-vitro antibacterial activity of common antibiotics and herb extracts to clinical isolates of Escherichia coli collected from UTI patient. International Journal of Research in Pharmaceutical and Biomedical Sciences, 3, (2), 987-992 [Link]

Hoerlle, J.L., Brandelli, A. (2009). Antimicrobial resistance of Staphylococcus aureus isolated from the intensive care unit of a general hospital in southern Brazil. Journal of Infection in Developing Countries, 3(7), 504510. [DOI]

Islam, M.R., Ahamed, R., Rahman, M.O., Akbar, M.A., Al-Amin, M. Alam, K.D., Lyzu, F. (2010). In-vitro antimicrobial activities of four medicinally important plants in Bangladesh. European Journal of Scientific Research, 39, (2), 199-206. [DOI]

Jeyachandran, R., Baskaran, X., Cindrella, L. (2010). In-vitro Antibacterial activity of three Indian medicinal plants. International Journal of Biological Technology, 1(1):103-106. [Link]

Khan, A., Rahman, M. Islam, S. (2007). Antibacterial, Antifungal and cytotoxic activities of tuberous roots of Amorphophallus campanulatus, Turkish Journal of Biology, 31, 167-172. [Link]

Kruiswijk, B. (2005). Thin layer Chromatography Visualization. (2nd Edition. pp:1-21.) West monmoth.

Kumar, R., Yadav, B.R., Singh, R.S. (2010). Genetic determinants of antibiotic resistance in Staphylococcus aureus isolates from milk of mastitic crossbred cattle. Current Microbiology, 60, 379-386. [DOI]
Locksley, R.M., Cohen, M.I, Quinn, T.C., Tompkins, L.S., Coyle, M.B. Kirihara, J.M., Counts. (1982). Multiple antibiotic resistant Staphylococcus aureus: Introduction, transmission and evolution of nosocomial infection. Annals of internal medicine, 9,317 - 324. [DOI]

Maity, P., Hansda, D., Bandyopadhyay, U., Mishra, K.D (2009). Biological activities of crude extracts and chemical constituents of Bael, Aegle marmelos (L). Indian Journal of Experimental Biology, 47, 849-861. [Link]

Masuduzzaman S., Meah M. B., Rashid M. M. (2008). Determination of Inhibitory Action of Allamanda Leaf Extracts Against Some Important Plant Pathogens Journal of Agriculture and Rural Development, 6(1\&2), 107-112. [DOI]

Mišan, A.Č., Mimica-Dukić N.M., Mandić, A.I., Sakač, M.B., Milovanović, I.L. ivana J. Sedej, I.J. (2011). Development of a rapid resolution HPLC method for the separation and determination of 17 phenolic compounds in crude plant extracts. Central European Journal of Chemistry, 9, (1), 133-142. [DOI]

Narmeen, S. Jaladet, M., Jubrael. M. S. (2009). Isolation and identification of Staphylococcus aureus using classical and molecular methods. Journal of Duhok University, 12, (1), 10-16. [Link]

Patra, J.K., Gouda, S., Sahoo, S.K., Thatoi, H.N. (2012). Chromatography separation, H NMR analysis and bioautography screening of methanol extract of Excoecaria agallocha L. from Bhitarkanika, Orissa, India . Asian Pacific Journal of Tropical Biomedicine, S50-S56.

Sasidharan, S. Chen, Y., Saravanan, D., Sundram, K.M., Latha, Y.L. 2011. Extraction, isolation and characterization of bioactive compounds from plants' extracts. African Journal of Traditional Complementary and Alternative Medicine, 8(1), 1-10. [DOI]

Sharmeen, R. Hossain, M.N., Rahman, M.M., Foysal, M.J., Miah, M.F. 2012 In-vitro antibacterial activity of herbal aqueous extract against multidrug resistant Klebsiella sp. isolated from human clinical samples. International Current Pharmaceutical Journal, 1(6), 133-137. [DOI]

Singh, P., Prakash, A. (2008). Isolation of Escherichia Coli, Staphylococcus Aureus And Listeria Monocytogenes. from milk products sold under market conditions at Agra region. Acta Agriculturae Slovenica, 92, (1), 83-88. [Link] 